\title{
Positron Emission Tomography and Computed Tomography Scan
}

National Cancer Institute

\section{Source}

National Cancer Institute. Positron Emission Tomography and Computed Tomography

Scan. NCI Thesaurus. Code C103512.

An imaging technique that utilizes positron emission tomography and computed tomography in a single machine. 